\title{
BMJ Open Public health implications of molecular point-of-care testing for chlamydia and gonorrhoea in remote primary care services in Australia: a qualitative study
}

\author{
L Natoli, ${ }^{1,2}$ R J Guy, ${ }^{1}$ M Shephard, ${ }^{3}$ D Whiley, ${ }^{4}$ S N Tabrizi, ${ }^{5}$ J Ward, ${ }^{6}$ D G Regan, ${ }^{1}$ \\ $\mathrm{S}$ G Badman, ${ }^{1} \mathrm{D}$ A Anderson, ${ }^{2} \mathrm{~J}$ Kaldor, ${ }^{1} \mathrm{~L}$ Maher, ${ }^{1}$ on behalf of the TTANGO \\ investigator group
}

To cite: Natoli L, Guy RJ, Shephard M, et al. Public health implications of molecular point-of-care testing for chlamydia and gonorrhoea in remote primary care services in Australia: a qualitative study. BMJ Open 2015;5:e006922. doi:10.1136/bmjopen-2014006922

- Prepublication history for this paper is available online. To view these files please visit the journal online (http://dx.doi.org/10.1136/ bmjopen-2014-006922).

Received 15 October 2014 Revised 13 March 2015 Accepted 20 March 2015

CrossMark

For numbered affiliations see end of article.

Correspondence to

L Natoli;

lisan@burnet.edu.au

\section{ABSTRACT}

Objectives: With accurate molecular tests now available for diagnosis of chlamydia and gonorrhoea (Chlamydia trachomatis (CT)/Neisseria gonorrhoeae (NG)) at the point-of-care (POC), we aimed to explore the public health implications (benefits and barriers) of their integration into remote primary care in Australia.

Methods: Qualitative interviews were conducted with a purposively selected group of 18 key informants reflecting sexual health, primary care, remote Aboriginal health and laboratory expertise.

Results: Participants believed that POC testing may decrease community prevalence of sexually transmitted infections (STIs), and associated morbidity by reducing the time to treatment and infectious period and expediting partner notification. Also, POC testing could improve acceptability of STI testing, increase testing coverage and result in more targeted prescribing, thereby minimising the risk of antibiotic resistance. Conversely, some felt the immediacy of diagnosis could deter certain young people from being tested. Participants also noted that POC testing may reduce the completeness of communicable disease surveillance data given the current dependence on reporting from pathology laboratories. Others expressed concern about the need to maintain and improve the flow of NG antibiotic sensitivity data, already compromised by the shift to nucleic acid-based testing. This is particularly relevant to remote areas where culture viability is problematic.

Conclusions: Results indicate a high level of support from clinicians and public health practitioners for wider access to CT/NG POC tests citing potential benefits, including earlier, more accurate treatment decisions and reductions in ongoing transmission. However, the data also highlight the need for new systems to avoid adverse impact on disease surveillance.

Trial registration number: Australian and New Zealand Clinical Trials Registry: ACTRN12613000808741.

\section{BACKGROUND}

A key public health strategy for the control of Chlamydia trachomatis (CT) and Neisseria

\section{Strengths and limitations of this study}

- The main strength of this study is that findings provide practical considerations to inform the responsible introduction of Chlamydia trachomatis (CT)/Neisseria gonorrhoeae (NG) point-ofcare $(\mathrm{POC})$ testing in remote services.

- To our knowledge, this is the first study to explore the public health implications of using CT/NG POC testing in a remote setting in Australia or elsewhere.

- The study is limited by the sample size (18 interviews); however, participant recruitment continued to the point of data saturation and we sampled to achieve diversity in demographics, expertise and jurisdiction.

- Qualitative methods are well suited to openended enquiry or exploratory research where little is known about the phenomena under study -and many of the issues identified here may have been missed if quantitative methods had been used.

gonorrhoeae $(\mathrm{NG})$ is the provision of accurate testing and timely treatment through primary care services. The objective of treatment is cure for those infected and prevention of onward transmission, and with high enough coverage, ultimately a reduction in prevalence of sexually transmitted infections (STIs) in the population. ${ }^{1}$

High rates of STIs exist in many remote areas in Australia, ${ }^{2}$ but despite efforts to increase the coverage of STI testing and treatment via primary care, patients in these remote communities may still experience substantial delays in receiving treatment. ${ }^{3}$ While 'syndromic management' is utilised for those with symptoms or considered to be at risk, it has poor sensitivity and specificity especially for women, leaving many infections untreated, and resulting in 
morbidity and further transmission as well as substantial overtreatment. ${ }^{45}$

A rapid point-of-care (POC) assay for STIs could theoretically increase the uptake and timeliness of treatment and reduce the average duration of infectiousness. ${ }^{6}$ Mathematical modelling suggests that a CT/NG POC test, even with moderate sensitivity, may be a useful public health intervention, especially in settings where there is strong potential for ongoing STI transmission in the time between testing and treatment, and where the likelihood of patients returning for STI test results and treatment is low. ${ }^{7}$ A POC test with high sensitivity $(>95 \%)$ was recently predicted by Hui $e t a l$ to reduce the prevalence of CT and NG from $11.9 \%$ to $8.9 \%$ and $7.1 \%$ to $5.7 \%$, respectively, when used in high prevalence settings. If annual STI screening coverage was increased, this impact would be further enhanced.

Testing for CT and NG at the POC may improve STI control in remote Australian communities and result in other public health benefits. For example, a clinical trial of syphilis POC tests in a rural antenatal setting in South Africa reduced the average time to treatment by 16 days. ${ }^{9}$ There have also been several modelling studies which have focused on the likely benefits (such as cost savings, and more accurate diagnosis and treatment) of using $\mathrm{CT} / \mathrm{NG} \mathrm{POC}$ tests in an urban clinical context. ${ }^{10}{ }^{11}$ Conversely, there may be barriers or unintended negative consequences associated with introducing STI POC testing. ${ }^{12}$ To our knowledge, there have been no studies which have comprehensively explored the potential public health benefits and challenges of using CT/NG POC tests. These issues need to be understood in order to support the routine integration of this testing in remote communities in Australia and other settings.

The Xpert CT/NG assay is the first rapid, portable, molecular diagnostic system suited to use at the POC, and has sensitivity and specificity ${ }^{13} 14$ similar to conventional nucleic acid laboratory-based tests. This assay is being utilised in the TTANGO (Test, Treat, ANd GO) trial, ${ }^{15}$ a cross-over cluster randomised controlled trial underway in 12 remote Australian communities where many Aboriginal and Torres Strait Islander Australians (hereafter referred to as Aboriginal) reside, and rates of NG and CT are high. ${ }^{2}$ The trial is the first to use a POC molecular diagnostic test for STIs in a primary healthcare setting anywhere in the world. ${ }^{15}$ The objective of this qualitative research, in the context of the TTANGO trial, was to explore the public health implications (both benefits and challenges) associated with $\mathrm{CT} / \mathrm{NG}$ POC test usage in remote primary care in Australia.

\section{METHODS}

Setting

Healthcare in remote Aboriginal communities is mainly provided through primary healthcare services, with the majority of clients $(>90 \%)$ being Aboriginal. Services utilise syndromic management with patients who are symptomatic or at risk, in addition to sending specimens to laboratories for testing, with asymptomatic patients treated if positive results are received. Many healthcare services are located hundreds to thousands of kilometres away from laboratories; specimen transport may only occur once a week ${ }^{16}$ and results may not be received from laboratories for a further 7-10 days, after which patients need to be recalled for follow-up. In some areas, due to client mobility and difficulties recalling patients, $11-25 \%$ of people remain untreated for diagnosed STIs and the average time to treatment is 21 days for asymptomatic patients, ${ }^{3}$ compared with 3 days in urban settings. ${ }^{17}$ While processes vary across jurisdictions, clinicians and laboratories are required to notify positive CT and NG results to state health departments for disease surveillance. ${ }^{18}$ Also, the National Neisseria Network of reference laboratories collaborate to monitor clinical isolates of pathogenic Neisseria species for antibiotic susceptibility and data are collated by the Australian Gonococcal Surveillance Program. ${ }^{19}$

\section{Study design}

Between March and August 2013, we conducted in-depth qualitative interviews with key informants to explore, among other topics, the public health implications (benefits and challenges) of integrating CT/NG POC testing into remote settings. Interviews focused on POC testing generally (not limited to molecular tests), though molecular tests were often the focus of discussion. An interview guide was developed, based on a review of the literature, in order to enable exploration of previously noted benefits and barriers to the integration of STI POC testing in remote practice. Questions were phrased in a non-leading manner to reduce interviewer bias. Where new and interesting issues were raised, they were further explored. Within a lengthier question guide that explored other issues, the following questions were central to the objective of this study to explore the public health benefits and barriers associated with CT/NG POC testing in remote primary care:

1. Can you tell me what you know about POC tests for chlamydia and gonorrhoea?

2. Can you tell us about any experiences you have had with POC tests more generally?

3. Are there any lessons from the use of other POC tests that should be taken in to account when implementing POC testing for chlamydia and gonorrhoea?

4. What are your views on the potential benefits to providing chlamydia and gonorrhoea POC tests for people living in remote areas in Australia?

5. What are your views on the potential barriers to providing chlamydia and gonorrhoea POC tests for people living in remote areas in Australia?

\section{Participants}

Purposive sampling was used to provide diversity in demographics and expertise. Participants were drawn from six 


\begin{tabular}{lc} 
Table 1 Participant background & n (\%) \\
\hline \multicolumn{2}{l}{ Australian jurisdiction where currently employed } \\
Queensland & $2(11)$ \\
New South Wales & $5(28)$ \\
Victoria & $4(22)$ \\
Northern Territory & $2(11)$ \\
Australian Capital Territory & $1(6)$ \\
Western Australia & $4(22)$ \\
Professional expertise & $8(44)$ \\
STI clinician (medical doctors and nurses) & $4(22)$ \\
Laboratory/microbiology & $4(22)$ \\
Policy and advocacy & $2(11)$ \\
Research & \\
Clinical or laboratory experience in remote Aboriginal \\
communities & $10(56)$ \\
Yes & $8(44)$ \\
No &
\end{tabular}

of the eight Australian States and Territories, and included individuals with current or previous professional experience (clinical or laboratory) in remote Aboriginal communities (56\%). In addition, we included other participants with knowledge of CT/NG POC tests or experience using POC tests for other STIs (table 1).

Participants $(n=18)$ included doctors (both primary care doctors with an interest in sexual health as well as those with specialist qualifications) and nurses working in urban, regional and remote health services; researchers; those working in sexual health policy and advocacy; and laboratory-based microbiologists. The median age of participants was 49 years (range $39-58$ years).

\section{Interviews}

Interviews were conducted by the primary author (LN) in person, if possible, and otherwise via telephone or internet, and took between 30 and $75 \mathrm{~min}$. Written informed consent was obtained from all participants.

\section{Data management and analysis}

Recruitment continued until the data were saturated or no new themes emerged. ${ }^{20}$ Interviews were digitally recorded, transcribed verbatim, and transcripts were checked for accuracy against the recordings and to ensure familiarisation prior to analysis. Transcripts were then uploaded into QSR Nvivo (V.10), a qualitative data management and analysis programme (QRS International PTY Ltd, Melbourne, Australia). Each transcript was systematically coded and content analysis was performed to examine frequencies of recurring codes and to identify salient themes. ${ }^{21}$

\section{RESULTS}

Interview participants identified a range of potential public health implications relating to the introduction of CT/NG POC testing in remote communities. These are summarised thematically in table 2 and detailed below.

\section{STI control}

Improving the timeliness of testing and treatment was recognised to be a key strategy to reduce population prevalence.

$[\mathrm{Y}]$ ou should be able to find someone, treat them and take them out of the infected pool. (Participant \#2).

The main reasons identified by participants for delays in treatment were patient mobility and distance of laboratories from clinics, combined with the wait for test results. POC testing was considered a means to overcome barriers to timely treatment.

\section{It's always been the challenge...breaking of the cycle of infection...you need to be able to test and treat people within a short...time period... and contact tracing...[If] you waited for people's results for two weeks...people can be a long way away from where they have the test, and if someone has an infection, this is a huge challenge... So I think that testing and treating onsite is marvellous. (Participant \#9)}

Many participants also noted that POC testing would allow staff to start partner notification more quickly.

[I]f you're talking about getting the result back in a week or two...often...people would have moved elsewhere and then trying to do the contact tracing, their partner may have moved elsewhere. So...I think it [POC testing] would be a huge benefit...reducing your time to treating both. (Participant \#15)

\section{Reduced morbidity from untreated STIs}

Reducing the time to treatment would in turn reduce the risk of complications from untreated STIs. This was noted by many respondents, who emphasised the longer term sequelae of undiagnosed chlamydia.

[W] ell the individual health benefits, particularly in women around long term undiagnosed chlamydia, so the implications around fertility. (Participant \#11)

\section{More targeted treatment}

Some felt that POC testing would result in more targeted prescribing and this, in turn, may have broader public health benefits such as mitigating antibiotic resistance.

I think in remote areas, if you do the test and it's just chlamydia, then you can spare anything that might be driving gonococcal resistance. You...don't have to treat them with the penicillin or ceftriaxone...that's actually very useful-rather than having to give everybody everything for everything. (Participant \#2) 
Table 2 Implications of CT/NG POC testing, summary of themes

Benefit Challenge

STI control

Decrease time between diagnosis and treatment, more timely partner notification

Decrease period of infectiousness, transmission, infectious pool and prevalence

Reduced morbidity from untreated STIs

Reduced complications (such as pelvic infertility disease and infertility) from untreated STIs

More targeted treatment

More targeted prescribing/reduced overprescribing

Potential to mitigate antibiotic resistance

Testing coverage

Potential to normalise testing and increase testing coverage

Potential use in outbreak settings

Immediacy of POC result may be a barrier to testing in some circumstances

Need to address existing barriers to STI testing

Prevalence and test performance

Influence of POC test sensitivity/specificity and prevalence on positive predictive value and negative predictive value

Health communication

Need for community engagement and health communication strategy

Disease surveillance

Potential to reduce completeness of chlamydia and gonorrhoea notification data

Monitoring of NG antibiotic sensitivity

Need for molecular antibiotic sensitivity surveillance methods

CT, Chlamydia trachomatis; NG, Neisseria gonorrhoeae; POC, point-of-care; STI, sexually transmitted infection.

In addition, having a definitive test result-as opposed to treating on the basis of symptoms (which lacks the specificity of laboratory tests) - might avert unnecessary notification of partners and potential social consequences such as violence.

[I]f you can establish they're negative for chlamydia and gonorrhoea, then there's much less pressure to initiate partner treatment...so it would avoid some unnecessarily damaging conversations and so on. (Participant \#6)

\section{Testing coverage}

Many respondents noted that increasing access to testing and achieving high community testing coverage are important public health outcomes. Several participants felt POC testing might lead to greater coverage by improving community acceptability of testing.

It might normalise testing and increase the acceptability of it...it would definitely increase the number of people who you would be able to test, especially the young ones who don't usually come into the clinic. (Participant \#1)

One participant felt that POC testing may provide an opportunity for increased patient engagement and the opportunity to test for other STIs.

[W] hen there is a positive result it does provide an opportunity to have a more frank discussion about what else is going on in people's lives. And then the opportunity to test for other...STIs like HIV that you otherwise would not have done. (Participant \#11)
At the same time, the immediacy of the POC test result was considered a potential barrier to testing by several respondents, particularly in relation to the testing of young people.

[S] ome young people may...feel a bit worried about getting an immediate diagnosis...young people can...feel a bit concerned and in some ways, they don't want to know 'just yet'. (Participant \#7)

Others noted a number of pre-existing barriers to STI testing which need to be addressed if high levels of POC test coverage are to be achieved.

[W]ith some of the best tools in the world it [opportunistic testing] doesn't happen to ideal levels. And that's about health worker comfort more than anything else. So the technology might be available, effective, specific, cost effective, but you've got some ...Aboriginal cultural but also health system cultural factors to deal with... (Participant \#3)

Achieving high testing coverage is particularly important in the context of outbreak control and POC tests may be useful in such circumstances. One participant explained their experience in relation to syphilis control.

[W]e've used it [syphilis POC test]...in circumstances where an immediate result was important to know,...we were happy to accept an element of overtreatment, but wanted to be able to do the treatment and contact tracing at the point of testing...So we're using it in an 
outbreak setting, and...it dramatically reduced the amount of staff time required to do the same amount of work, but it also was very popular with the patients. (Participant \#12)

\section{Prevalence and test performance}

Participants emphasised the importance of positive and negative predictive value, and how this would be influenced by STI prevalence. A POC test would require sensitivity and specificity as close as that achievable from laboratory tests, if such test results are to instil confidence in health staff, inform clinical decision-making and enable realisation of the many public health benefits noted above. Participants expressed concerns about the potential for false-positive results and highlighted the negative consequences of such results.

[I]f you've got a...non-Aboriginal young heterosexual population, where the prevalence is...it's going to range from 3 to $5 \%$, that's relatively low prevalence. So if you've got a test that doesn't have a fantastic sensitivity or specificity, you're going to get really high false positive rates, and the psychosocial consequences of giving a 19 or 17 year old a Chlamydia diagnosis when they don't have it can be...dire. (Participant \#8)

[I] $\mathrm{n}$ an Aboriginal community...the consequence of a positive result often has to be handled...more sensitively...than in non Aboriginal settings...a positive test within a partnership, you know a perceived marriage relationship can be very stressful...it can...you know threaten and make unsafe conditions for people, women in particular. (Participant \#3)

\section{Health communication}

Several participants indicated that focused communication and engagement should precede POC testing programmes to ensure the community is well informed and trusts the technology.

[O]ver the next 5-10 years it's going to be really important that...informed experts are able to get the message out into the community...that performance of these tests is very good and...reliable. (Participant \#11)

At the same time, it was noted that health communication when first introduced may have a negative effect on attendance for testing.

[W] e need to be cognisant that people...may be reluctant to go to a service...when you've just hammered a community around 'hey look what's available'- you know, then you don't want to necessarily...be [seen] there first thing Monday morning. (Participant \#11)

\section{Disease surveillance}

Participants also expressed concerns about the potential impact of POC testing on disease surveillance and completeness of notification data, which is largely based on reports from diagnostic laboratories.
It [surveillance] is about...comparing numbers in Aboriginal vs non Aboriginal settings to see if our programs are working, it's about getting the demographic breakdown, males and females, so we'd miss all that...it's also about knowing the denominator and knowing how many people are being tested... and how would we monitor that if we weren't getting some kind of record from the clinics? (Participant \#14)

Several participants mentioned that notification based on laboratory reporting is more reliable than clinic or clinician-based reporting, and voiced concern about how the surveillance picture may change with a shift to POC testing.

At the moment we have automatic laboratory notification that goes to the health department, so it doesn't rely on the clinician filling out a notifiable disease form... If you run a [POC test] system in a clinic and they're not very good at following up on their notifications then you're going to lose that data and I think the health department would not be very happy. (Participant \#17)

\section{Monitoring of NG antibiotic sensitivity}

Many participants acknowledged that 'in theory' POC testing could lead to there being no culture-viable isolates available for NG antibiotic sensitivity surveillance. However, it was recognised that in remote areas, due to the widespread shift to nucleic acid amplification test (NAAT) and urine collection instead of swabs, there has been a reduction in the number of isolates available for culture anyway.

[S] ince PCR has been used there's hardly anyone does any cultures for gono [gonorrhoea]...even if they are, your chances from a lot of those remote settings in growing anything is minimal...[I]n practice I honestly don't think it [POC testing] will really make any difference in a remote setting. (Participant \#15)

Several participants highlighted that rather than continuing with culture-based monitoring of antibiotic sensitivity in remote Australia, nucleic acid-based methods should be adapted to provide resistance profiles instead.

$[\mathrm{M}]$ olecular surveillance is the way to go, rather than putting out...claims we should culture more...it's not going to happen in these remote regions, when you have one pick up a week collecting your specimens from some of these communities...Molecular diagnosis is...going to become more prominent, there will always be some cultures which is good but it's representativeness for what is going on out there in the real world is diminishing. (Participant \#17)

Continuing to monitor NG antibiotic sensitivity was highlighted to be especially critical in some remote areas, where resistance profiles of NG infections may vary.

[W] here there is a combination of Indigenous Australian infections plus...fly in-fly out miners...it's really 
important that we get ideal specimens but we also get the capability to do resistance testing. Because if we miss that then we have the potential for all of the benefits of point of care testing to be washed away through inappropriate management. (Participant \#18)

\section{DISCUSSION}

This study has highlighted a range of potential public health implications associated with the introduction of CT/NG POC testing in remote Australia. While stakeholders recognised important public health benefits of this innovative technology, they also identified challenges that need to be considered before routine implementation.

The public health benefits identified included reducing the duration of infection through prompt treatment and partner notification, reducing prevalence and STI morbidity, more targeted prescribing that may reduce overtreatment and mitigate the development of antibiotic resistance, and increasing testing coverage by enhancing acceptability of STI testing. Many of these benefits have been evaluated in mathematical modelling studies, ${ }^{8} 101122$ but there is lack of empirical data to confirm this impact.

Participants also recognised that the integration of POC testing is not without challenges. ${ }^{23}{ }^{24}$ Some participants questioned whether testing coverage would actually increase, given the existing cultural and health service barriers to STI testing. ${ }^{25}{ }^{26}$ As estimated recently, CT and NG POC tests are likely to have the greatest public health benefit in high prevalence areas if coupled with increased testing coverage. ${ }^{8}$ Thus, any programme introducing POC testing should also work with services to address barriers to STI testing generally.

Participants were also concerned that testing coverage might decrease due to patients' apprehension regarding the immediacy of the results. Similar theoretical concerns were raised in Australia before the introduction of HIV rapid tests, but these have not materialised in practice. $^{27}$

Some participants had concerns about the positive and negative predictive value of POC tests and how this would be influenced by STI prevalence. When selecting a POC test, the test sensitivity and specificity along with the STI disease prevalence in the population are important considerations. In the case of the GeneXpert, the test performance is similar to routine NAATs, ${ }^{13} 14$ but the additional two gonorrhoeae targets in GeneXpert mean the test can undertake screening and confirmation simultaneously, and thus substantially reduce the chance of false-positive results even in low prevalence areas.

Another issue identified by participants was the potential impact of POC testing on disease surveillance processes. In Australia, in jurisdictions that require STI notification from both clinicians and laboratories, nearly half are made by laboratories alone, ${ }^{28}$ probably because laboratories have a larger volume of positive results and have automated electronic report procedures. Thus, if notification becomes the responsibility of the clinic, automated systems may be needed. This is entirely possible, as POC connectivity software is becoming increasingly sophisticated. Collation of both positive and negative results would enable calculation of STI positivity, a useful prevention indicator. ${ }^{29}$ Only a small number of Australian health departments collate negative test results (as well as positives) as negative results are not a notifiable condition; thus, health departments need to establish additional systems in collaboration with laboratories.

The impact of POC testing on NG antibiotic resistance monitoring was also raised. Ensuring these systems are retained and improved is vital considering global concerns about the threat of NG becoming untreatable. ${ }^{30}$ Obtaining viable samples for culture of gonorrhoeae isolates is particularly challenging in remote Australia due to vast distances between clinics and reference laboratories, and environmental conditions. The flow of NG sensitivity data has also been compromised by the increasing shift to urine collection and NAAT, and strategies are needed to manage this before any widespread shift to the POC approach. Participants noted the potential utility of molecular methods for non-culture-based NG antimicrobial resistance surveillance; ${ }^{31}$ a programme of PCR-based tracking of penicillinase-producing NG is already underway in Western Australia. ${ }^{32}$ Systems could be established whereby a positive POC test (on either urine or swab) triggers forwarding of the urine specimen or collection of an additional swab for molecular monitoring of markers of resistance. It may also be possible for PCR markers for NG resistance to be integrated into POC tests, as is the case for rifampicin resistance in the corresponding Xpert test for Mycobacterium tuberculosis.

Our study has several limitations. The qualitative approach, small sample size, and the non-random nature of the sampling strategy limit the generalisability of the results. However, as we purposely sampled with a broad range of recognised experts from different disciplines and jurisdictions with expertise in the testing and diagnosis of STIs, the volume of data generated was substantial, as was the depth and detail of the interview transcripts. Coding of the interview transcripts by one author may result in a biased interpretation of the data.

In conclusion, Australian stakeholders firmly believe that molecular CT/NG POC tests can improve STI management and control in high prevalence remote areas. Modelling shows that an increase in testing coverage is needed to maximise benefit, but there were mixed views from stakeholders if this could be achieved by POC testing alone. The interviews highlighted the need to address the public health aspects of POC testing, including development of communication plans and strategies to enhance communicable disease and NG antimicrobial resistance surveillance systems. There is also a need for further trials to assess if POC testing can increase both testing and treatment coverage, and reduce community STI prevalence in high prevalence settings. 
Author affiliations

${ }^{1}$ The Kirby Institute, University of New South Wales, Sydney, New South Wales, Australia

${ }^{2}$ Burnet Institute, Melbourne, Victoria, Australia

${ }^{3}$ Flinders University International Centre for Point of-Care Testing, Flinders University, Adelaide, South Australia, Australia

${ }^{4}$ Queensland Paediatric Infectious Diseases (QPID) Laboratory, Queensland Children's Medical Research Institute, The University of Queensland, Brisbane, Queensland, Australia

${ }^{5}$ Department of Microbiology and Infectious Diseases, The Royal Women's Hospital, Melbourne, Victoria, Australia

${ }^{6}$ South Australian Health and Medical Research Institute, Adelaide, South Australia, Australia

Acknowledgements The authors would like to acknowledge and thank the interview participants who gave us generously some of their time. The authors gratefully acknowledge the contribution to this work of the Victorian Operational Infrastructure Support Program received from the Burnet Institute.

Collaborators Test, Treat and Go (TTANGO) Investigators include RJG, JK, Basil Donovan, David Wilson, Handan Wand, DGR, SGB and Louise Causer from the Kirby Institute, JW (Baker IDI), Belinda Hengel (Apunipima Health Council), Annie Tangey (Ngaanyatjarra Health Service), LN and DAA (Burnet Institute), DW (The Queensland Pediatric Infectious Diseases Laboratory), SNT (The Royal Women's Hospital), MS (Flinders University), and Christopher Fairley (Monash University Central Clinical School and Melbourne Sexual Health Centre). There are a number of collaborators critical to the trial's success and these include: participating health services; the TTANGO Reference Group; Queensland Aboriginal and Islander Health Council; Queensland Health; Aboriginal Health Council of Western Australia; West Australia Department of Health; West Australian Country Health Service; Aboriginal Health Council of South Australia; South Australia Health; Western Diagnostic Pathology; PathWest; Clinipath Pathology; Pathology Queensland; Sullivan and Nicolaides Pathology; SA Pathology; National Reference Laboratory; Medical Communications Associates; and Cepheid.

Contributors LN performed data collection and analysis, and led the development, drafting and revision of the manuscript. RJG and LM advised on data collection tools, interview process and data analysis, and supported manuscript development and critical revision. MS, DW, SNT, DGR, SGB, JK, DAA and JW provided critical review of manuscript and revision of drafts. RJG provided overall supervision of manuscript. All authors read and approved the final paper.

Funding TTANGO is funded by a National Health and Medical Research Council (NHMRC) Project Grant \# 109902. The Kirby Institute receives funding from the Australian Government Department of Health and Ageing.

Competing interests Cepheid has provided GeneXpert machines on loan for the duration of the TTANGO Trial.

Ethics approval West Australian Aboriginal Health Information and Ethics Committee (WAAHIEC); West Australian Community Health Board Research Ethics Committee (WACHBREC); Townsville Health Service District Human Research Ethics Committee; Cairns Health Service District Human Research Ethics Committee; Aboriginal Health Research Ethics Committee of South Australia (AHCSA).

Provenance and peer review Not commissioned; externally peer reviewed.

Data sharing statement No additional data are available.

Open Access This is an Open Access article distributed in accordance with the Creative Commons Attribution Non Commercial (CC BY-NC 4.0) license, which permits others to distribute, remix, adapt, build upon this work noncommercially, and license their derivative works on different terms, provided the original work is properly cited and the use is non-commercial. See: http:// creativecommons.org/licenses/by-nc/4.0/

\section{REFERENCES}

1. WHO. Global strategy for the prevention and control of sexually transmitted infections: 2006-2015. Breaking the chain of transmission. Geneva: WHO, 2007.
2. Guy R, Garton L, Taylor Thomson D, et al. The 2010 baseline prevalence assessment conducted by the STRIVE trial. Australasian Sexual Health Conference. Canberra, Australia, 2011.

3. Guy R, Ward JS, Smith KS, et al. The impact of sexually transmissible infection programs in remote Aboriginal communities in Australia: a systematic review. Sex Health 2012;9:205-12.

4. Dallabetta GA, Gerbase AC, Holmes KK. Problems, solutions, and challenges in syndromic management of sexually transmitted diseases. Sex Transm Infect 1998;74(Suppl 1):S1-11.

5. Pettifor A, Walsh J, Wilkins V, et al. How effective is syndromic management of STDs? A review of current studies. Sex Transm Dis 2000;27:371-85.

6. Peeling RW. Applying new technologies for diagnosing sexually transmitted infections in resource-poor settings. Sex Transm Infect 2011;87(Suppl 2):ii28-30.

7. Vickerman $\mathrm{P}$, Watts $\mathrm{C}$, Alary $\mathrm{M}$, et al. Sensitivity requirements for the point of care diagnosis of Chlamydia trachomatis and Neisseria gonorrhoeae in women. Sex Transm Infect 2003;79:363-7.

8. Hui BB, Wilson DP, Ward JS, et al. The potential impact of new generation molecular point-of-care tests on gonorrhoea and chlamydia in a setting of high endemic prevalence. Sex Health 2013;10:348-56.

9. Myer L, Wilkinson D, Lombard C, et al. Impact of on-site testing for maternal syphilis on treatment delays, treatment rates, and perinatal mortality in rural South Africa: a randomised controlled trial. Sex Transm Infect 2003;79:208-13.

10. Turner KM, Round J, Horner P, et al. An early evaluation of clinical and economic costs and benefits of implementing point of care NAAT tests for Chlamydia trachomatis and Neisseria gonorrhoea in genitourinary medicine clinics in England. Sex Transm Infect 2014;90:104-11.

11. Adams EJ, Ehrlich A, Turner KM, et al. Mapping patient pathways and estimating resource use for point of care versus standard testing and treatment of chlamydia and gonorrhoea in genitourinary medicine clinics in the UK. BMJ Open 2014;4:e005322.

12. Pai NP, Vadnais C, Denkinger $C$, et al. Point-of-care testing for infectious diseases: diversity, complexity, and barriers in low- and middle-income countries. PLoS Med 2012;9:e1001306.

13. Tabrizi SN, Unemo M, Golparian D, et al. Analytical evaluation of GeneXpert CT/NG, the first genetic point-of-care assay for simultaneous detection of Neisseria gonorrhoeae and Chlamydia trachomatis. J Clin Microbiol 2013;51:1945-7.

14. Causer LM, Hengel B, Natoli L, et al. Field evaluation of three point-of-care tests for chlamydia and gonorrhoea in remote health services in Australia. STI and AIDS World Congress. Vienna, 2013.

15. Guy RJ, Natoli L, Ward J, et al. A randomised trial of point-of-care tests for chlamydia and gonorrhoea infections in remote Aboriginal communities: Test, Treat ANd GO- the "TTANGO" trial protocol. BMC Infect Dis 2013;13:485.

16. Shephard M. Point-of-care testing in Australia: the status, practical advantages, and benefits of community resiliency. Point Care 2013;12:41-5

17. Chen MY, Ryder N, Donovan B. Completeness and timeliness of treatment for chlamydia within a sexual health service. Int J STD AIDS 2004;15:762-4.

18. The Kirby Institute. HIV, viral hepatitis and sexually transmissible infections in Australia Annual Surveillance Report 2013. Sydney: The Kirby Institute, The University of New South Wales, 2013.

19. Lahra MM; Australian Gonococcal Surveillance Programme. Australian Gonococcal Surveillance Programme annual report 2012. Commun Dis Intell Q Rep 2013;37:E233-9.

20. Gibbs L, Kealy M, Willis K, et al. What have sampling and data collection got to do with good qualitative research? Aust N Z J Public Health 2007;31:540-4.

21. Pope C, Ziebland S, Mays N. Analysing qualitative data. BMJ 2000;320:114-16

22. Vickerman P, Watts C, Peeling RW, et al. Modelling the cost effectiveness of rapid point of care diagnostic tests for the control of HIV and other sexually transmitted infections among female sex workers. Sex Transm Infect 2006;82:403-12.

23. Peeling RW. Utilisation of rapid tests for sexually transmitted infections: promises and challenges. Open Infect Dis J 2009;3:156-63.

24. Peeling RW, Mabey D, Ballard RC. Introducing new diagnostics into STI control programmes: the importance of programme science. Sex Transm Infect 2013;89:115-19.

25. Hengel B, Jamil MS, Mein JK, et al. Outreach for chlamydia and gonorrhoea screening: a systematic review of strategies and outcomes. BMC Public Health 2013;13:1040.

26. Huang RL, Torzillo PJ, Hammond VA, et al. Epidemiology of sexually transmitted infections on the Anangu Pitjantjatjara Yankunytjatjara Lands: results of a comprehensive control program. Med J Aust 2008;189:442-5. 
27. Conway DP, Holt M, McNulty A, et al. Multi-centre evaluation of the Determine HIV Combo assay when used for point of care testing in a high risk clinic-based population. PLOS ONE 2014;9:e94062.

28. Department of Health Victoria. Infectious diseases notification trends and practices in Victoria, 2009. Vic Infect Dis Bull 2010;13:85-90.

29. Ali H, Guy RJ, Fairley CK, et al. Understanding trends in genital Chlamydia trachomatis can benefit from enhanced surveillance: findings from Australia. Sex Transm Infect 2012;88:552-7.

30. Whiley DM, Goire N, Lahra MM, et al. The ticking time bomb: escalating antibiotic resistance in Neisseria gonorrhoeae is a public health disaster in waiting. J Antimicrob Chemother 2012;67: 2059-61.

31. Goire N, Lahra MM, Chen M, et al. Molecular approaches to enhance surveillance of gonococcal antimicrobial resistance. Nat Rev Microbiol 2014;12:223-9.

32. Speers DJ, Fisk RE, Goire N, et al. Non-culture Neisseria gonorrhoeae molecular penicillinase production surveillance demonstrates the long-term success of empirical dual therapy and informs gonorrhoea management guidelines in a highly endemic setting. J Antimicrob Chemother 2014;69:1243-7. 